\title{
Influence of Structure Parameters and Velocity on Inflation Performance of Vortex Ring Parachute
}

\author{
Ma Xiaodonga ${ }^{\text {* }}$, Guo Ruib, Liu Rongzhong ${ }^{\mathrm{c}}$ and Lv Shengtao ${ }^{\mathrm{d}}$ \\ School of Mechanical Engineering, Nanjing University of Science \& Technology, Nanjing 210094, \\ Jiangsu, China \\ abqnj6222007@126.com, bguoruid@126.com, cliurongz116.com, dIvshengtao@gmail.com
}

Keywords: Vortex ring parachute; Inflation; Opening performance; Arbitrary Lagrange-Euler method; Fluid-structure interaction

\begin{abstract}
Based on arbitrary Lagrange-Euler method, a vortex ring parachute simulation model was set up, and its inflation was studied under the condition of infinite mass and low-speed airflow. Through changing line length, canopy area and airflow velocity, the inflation processes were simulated, and the time-histories curves of projective diameter and spinning rate were obtained. The influence of structure parameters and airflow velocity on parachute motion characteristics was analyzed. The results show that the limit spinning rate of vortex ring parachute increases with the difference increase of trailing-edge pitch line and leading-edge pitch line. With smaller canopy area and larger airflow velocity, the opening time and steady time of the parachute are shorter, and the limit spinning rate is higher, which is good for inflation and improving kinematic stability.
\end{abstract}

\section{Introduction}

Vortex ring parachute is a kind of common rotating parachute, who has advantages of big drag coefficient, low opening load, low cost and simple maintainance. So it is widely used in many fields, such as trajectory control of armament, deceleration and landing control of aircraft, airborne and deliver, recovery of aerospacecraft and outerspace exploration and landing, et al ${ }^{[1]}$.

During whole working process of parachute, inflation is the most significant stage ${ }^{[2]}$. At the same time, the physics course of the stage, involving fluid structure interaction and structural dynamics of transient large deformation, is most complicated, and the theoretical study is very difficult. In recent years, with the rapid development of computer technique, numerical simulation, which is economical and flexible, has been an effective method to study parachute inflation.

Most previous works of parachute inflation using FSI technique focus on circle flat parachute or cone parachute ${ }^{[3-9]}$. However, the inflation of rotating parachute is not paid enough attention to and remains to be explored. In this paper, based on the FSI method, the 3D numerical simulation of a vortex ring parachute is studied. The canopy shape change and the time-history curves of projective diameter and spinning rate are obtained. The accuracy of the simulation results is verified by experimental data. Then the effects of suspension line length, canopy area and airflow on inflation time, steady time and spinning rate are studied for the first time.

\section{Structure and numerical model}

Vortex ring parachute. Fig. 1 illustrates the inflated geometric model of vortex ring parachute, primarily composed of 4 canopies and 33 lines. Fig. 2 shows plane development of the parachute. The expanded view of canopy is heptagon plane with curve edges, and canopies are central symmetrical around the parachute axis.

Define line difference $\Delta L_{\mathrm{TL}}=L_{\mathrm{T}}-L_{\mathrm{L}}$, where $L_{\mathrm{T}}$ and $L_{\mathrm{L}}$ are the length of trailing-edge pitch line and leading-edge pitch line respectively. 


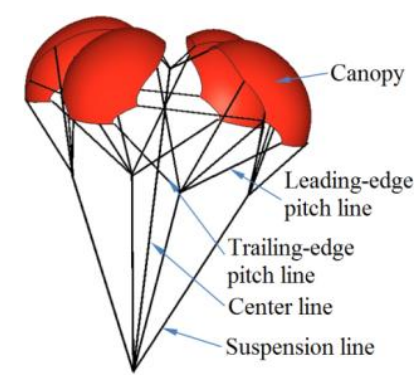

Fig. 1 Configuration of vortex ring parachute

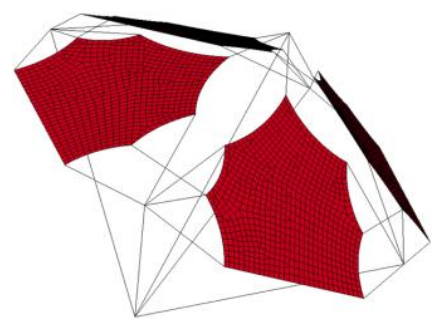

(a) Initial model

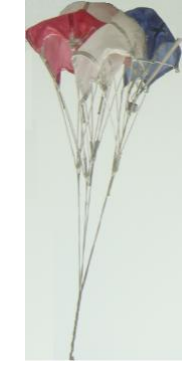

(b) Physical model

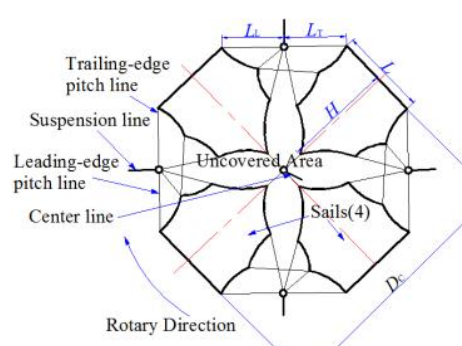

Fig. 2 Platform of vortex ring parachute
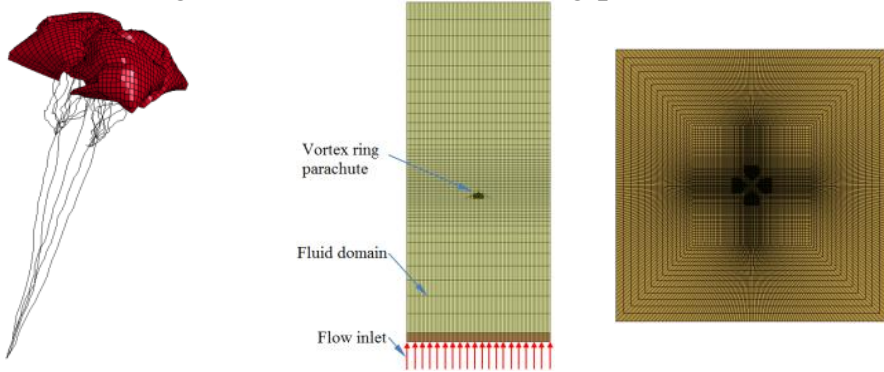

(d) Fluid domain model

Fig. 3 Finite element model of FSI

Numerical model. Fig. 3(a) shows the initial finite model of the vortex ring parachute. The canopy stays as an unfolded plane, which is meshed by 2892 shell elements. Some suspension lines stay in folded state ${ }^{[9]}$ and others in straightened state. Lines are meshed by 679 discrete cable elements and inner stress is zero. The intersection of the suspension lines and the center line is fixed, which the infinite quality condition is. 612128 hexahedral elements are used to mesh the flow field, whose inlet is a channel with a constant prescribed rate of air flow and other boundaries are non-reflecting, as shown in Fig. 3(d). The canopy permeability is considered.

To make the initial inflation model close to actual shape, the interaction of the model in Fig. 3(a) and the flow field is calculated firstly by ALE method ${ }^{[8]}$. When the parachute appearance turns similar to the fasciculation shape as shown in Fig. 3(b), restrict the motion of the parachute elements and export the finite model of the parachute. The finite model shown in Fig. 3(c) is taken as the initial inflation model, and then the interaction renew to be calculated from $t=0$.
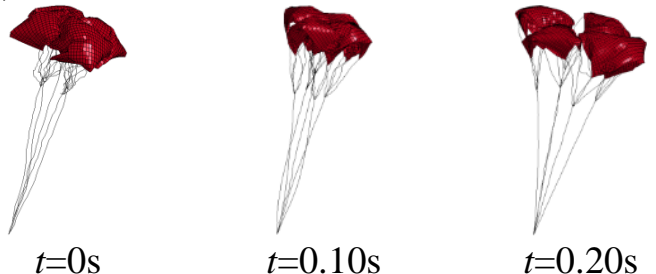

$$
t=0.20 \mathrm{~s}
$$

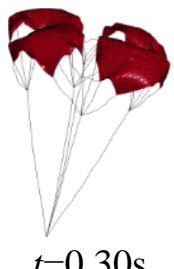

$t=0.30 \mathrm{~s}$

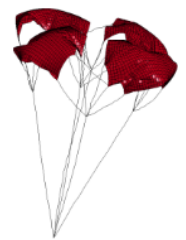

$t=0.50 \mathrm{~s}$

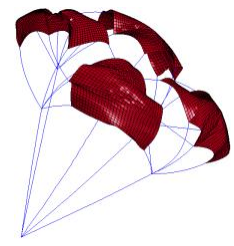

$t=1.00 \mathrm{~s}$

Fig. 4 Inflation of vortex ring parachute

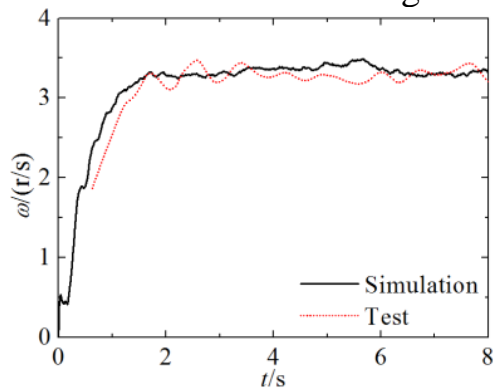

Fig. 5 Angular velocity

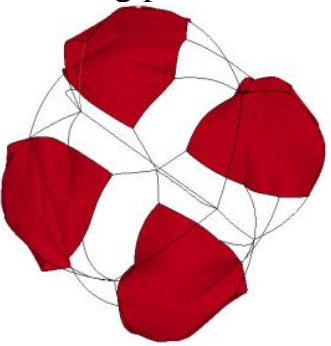

(a) Simulation

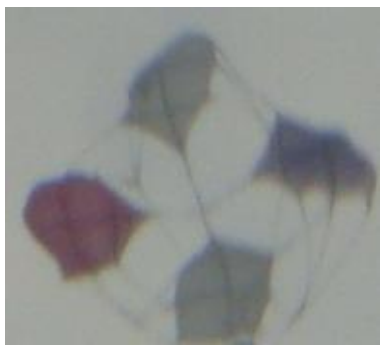

(b)Tower test

Fig. 6 The inflated canopy shape comparison

\section{Result analysis and discussion}

Inflation. The time-sequence parachute shape with the airflow velocity of $12 \mathrm{~m} / \mathrm{s}$ is shown in Fig. 4 . Fig. 5 shows the spinning rate comparison of simulation and test. In tower test ${ }^{[10]}$, the limit spinning rate of the model is about $3.30 \mathrm{r} / \mathrm{s}$ and fluctuates mildly. The vertical limit velocity is $11.02 \mathrm{~m} / \mathrm{s}$, and the 
error is $8.2 \%$. Fig. 6 illustrates the inflated shape comparison. The inflated shapes of simulation and test show a great agreement. Since there is crosswind in the air during the dropping process, the out edges of two canopies have relative larger deformation. Obviously, the tower test data shows a great agreement with the simulation results, and the initial inflation model has a good consistency with the initial state of the test model. Therefore, ALE method can simulate the inflation process of vortex ring parachute accurately.

Suspension line length effect on dynamic performance of vortex ring parachute. Keeping the canopy area invariant and changing the leading-edge and trailing-edge pitch line length, inflation processes of vortex ring parachute with different $\Delta L_{\mathrm{TL}}$ are obtained.

Fig. 7 and Fig. 8 show the projective diameters and spinning rates of vortex ring parachute with different $\Delta L_{\mathrm{TL}}$ respectively. Keeping $L_{\mathrm{L}}$ invariant and increasing $L_{\mathrm{T}}$, the limit spinning rate increases, which are $3.3 \mathrm{r} / \mathrm{s}, 3.5 \mathrm{r} / \mathrm{s}$ and $3.6 \mathrm{r} / \mathrm{s}$ respectively. This is because with bigger $\Delta L_{\mathrm{TL}}$, the convexity gradient of inflated canopy is larger, which makes the spinning torque coefficient greater. The time that the diameter comes to be a constant maximum increases, meaning that the parachute inflation time increases. But for the integral structures of the parachute are similar, the time differences are very minor.

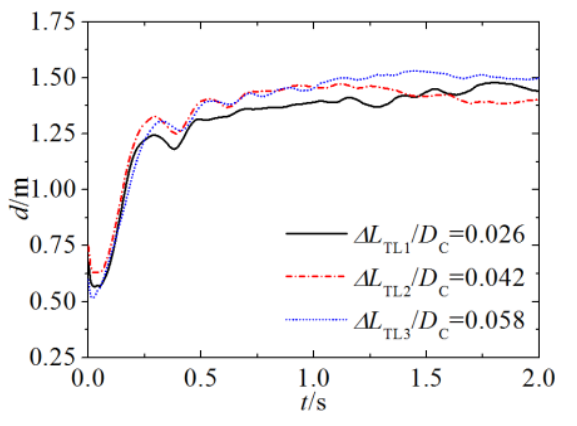

Fig. $7 d$ versus $\Delta L_{\mathrm{TL}}$

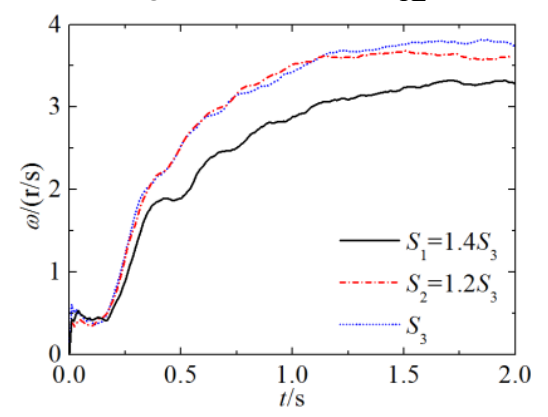

Fig. $10 \omega$ versus $S$

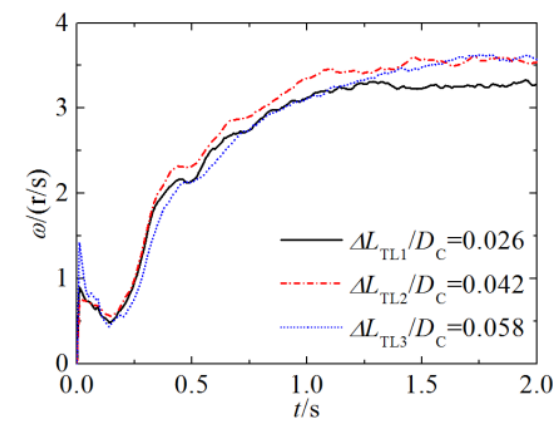

Fig. $8 \omega$ versus $\Delta L_{\mathrm{TL}}$

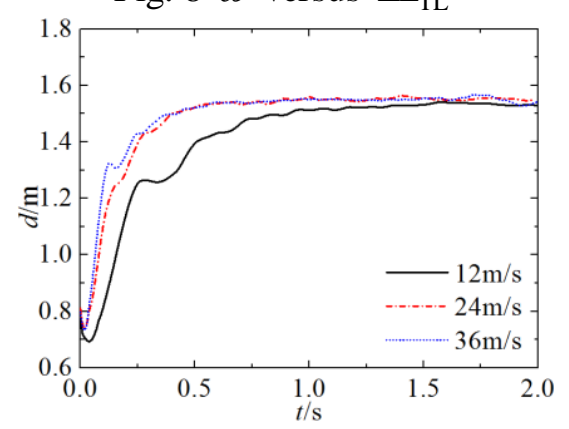

Fig. $11 d$ versus $v$

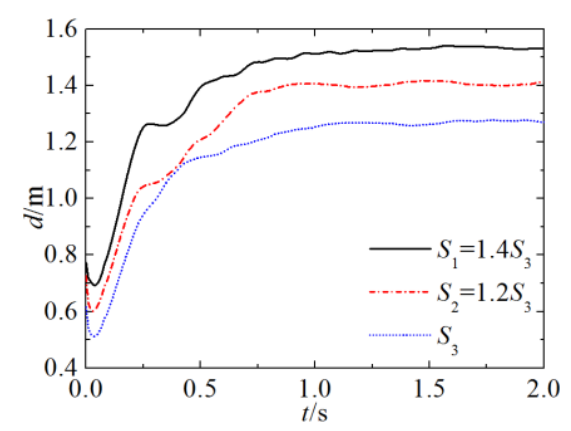

Fig. $9 d$ versus $S$

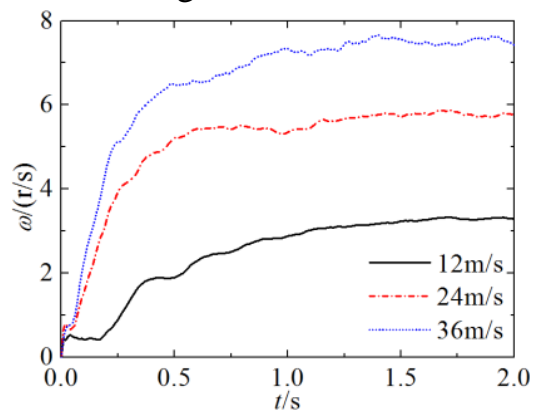

Fig. $12 \omega$ versus $v$

Canopy area effect on dynamic performance of vortex ring parachute. The canopy area has a close relation to the limit velocity of the parachute system with certain payload. The physical truth is that the limit velocity is higher as the area is smaller. At the same time, the limit spinning rate also varies with the area. Keeping the canopy structure shape invariant and changing its area, the inflation processes of vortex ring parachute with different canopy areas are obtained.

The inflation time becomes longer with the increasing area, as show in Fig. 9, verifying the conclusion in literature [2]: the bigger a parachute is, the longer the inflation time is. The limit spinning rate decreases with the increasing area, as shown in Fig. 10. Therefore, if a vortex ring parachute is requested to have a superior steady spinning rate and achieve inflation in a relatively shorter time, its canopy area should be smaller.

Velocity effect on dynamic performance of vortex ring parachute. To comprehend the effect of velocity on the dynamic performance, the inflation processes of vortex ring parachute under different airflow velocities, $v=12 \mathrm{~m} / \mathrm{s}, v=24 \mathrm{~m} / \mathrm{s}, v=36 \mathrm{~m} / \mathrm{s}$, are simulated. The computing results are stable in different velocity states.

The projective diameter is shown in Fig. 11. It can be found that the larger the velocity is, the faster the parachute inflates, leading to a shorter inflation time. As shown in Fig. 12, the numerical results 
show that the larger the velocity is, the higher the limit spinning rate of vortex ring parachute is. $\omega=3.30 \mathrm{r} / \mathrm{s}, \omega=5.80 \mathrm{~m} / \mathrm{s}$ and $\omega=7.40 \mathrm{~m} / \mathrm{s}$ are corresponding to $v=12 \mathrm{~m} / \mathrm{s}, \nu=24 \mathrm{~m} / \mathrm{s}$ and $v=36 \mathrm{~m} / \mathrm{s}$ separately. In ideal conditions, the rate $\omega / v$ for a vortex ring parachute is a constant. But when the airflow velocity increases, the mesh deformation becomes more serious, leading to that the inflated canopy gradient decreases. So the rate $\omega / v$ becomes smaller with a higher velocity.

\section{Conclusion}

(1) Through changing the length of suspension lines, the canopy inflated shape can be changed, making the spinning torque coefficient of vortex ring parachute vary. Consequently, the limit spinning rate value will be affected, so is the inflation time. Enhancing the length difference of leading-edge pitch and trail-edge pitch line, the steady spinning rate of vortex ring parachute will increase, and the inflation time will be longer.

(2) With smaller canopy area, vortex ring parachute will be inflated in a shorter time and the limit spinning rate will be higher. Therefore, canopy with relatively smaller area is benefit for inflation and improving kinetic stability.

(3) With the increase of the airflow velocity, the parachute inflation time becomes shorter and the limit spinning rate becomes higher.

\section{Acknowledgements}

This research was supported in part by National Natural Science Foundation of China(11102088), and Postgraduate Education and Innovation Project of Jiangsu Province(CXLX12-0210).

\section{References}

[1] R. Guo, R.Z. Liu. Dynamics model and simulation of the rigid and flexible coupling system for terminal-sensitive submunition. Acta Armament Arii 2007; 28(1):10-14.

[2] L.R. Wang. The theory and application of parachute. Peking: Aerospace press; 1997.

[3] K.R. Stein, R.J. Benney, E.C. Steeves. A computational model that couples aerodynamic and structural dynamic behavior of parachutes during the opening process. NASA-ADA-264115; 1993.

[4] T.E. Tezduyar, M. Behr, J. Liou. A new strategy for finite element computations involving moving boundaries and interfaces-the deforming-spatial-domain/space-time procedure: I.the concept and the preliminary numerical tests. Comput Methods Appl Mech Eng 1992; 94: 339-51.

[5] K. Takizawa, T.E. Tezduyar. Computational methods for parachute fluid-structure interactions. Arch Comput Methods Eng 2012; 19: 125-169.

[6] K. Yongsam, S. P. Charles. 3-D Parachute simulation by the immersed boundary method. Comput Fluids 2009; 38:1080-1090.

[7] J.D. Kim, Y. Li, X.L. Li. Simulation of parachute FSI using the front tracking method. J Fluids Struct 2009; 38:1080-90.

[8] N. Aquelet, B. Tutt. Euler-lagrange coupling for porous parachute canopy analysis. International J Multiphysics 2007; 1(1): 53-68.

[9] X.D. Ma, R.Z. Liu, Guo R, Zhang J. Simulation research on inflation of vortex rotating parachute system. Spacecr Remote Sens 2014; 34(2): 1-8.

[10]R. Guo, R.Z. Liu, Z.P. Hu, X.D. Ma. Study on the inflated stability and motional characteristic of vortex ring parachute canopy. Acta Aerodynamica Sin 2013; 31(6): 733-738. 